prevent outbreaks, and lower mortality rates, especially in this time of the SARS-CoV-2 pandemic. With overloaded health systems and shortages of health workers trained in infection management, as well as medical consumables and equipment, the best preventive measure remains changing gloves and hand washing.

Acknowledgments. The authors thank Dr Janet W. Reid for her English text review.

Financial support. This study was financed in part by the Coordenação de Aperfeiçoamento de Pessoal de Nível Superior, Brazil (CAPES), Finance Code 001. These government funds covered only the cost of laboratory materials and had no role in the study design or the decision to submit the work for publication.

Conflicts of interest. All authors report no conflicts of interest relevant to this article.

\section{References}

1. Grasselli G, Zangrillo A, Zanella A, et al. Baseline characteristics and outcomes of 1591 patients infected with SARS-CoV-2 admitted to ICUs of the Lombardy Region, Italy. JAMA 2020;323:1574-1581.
2. Lima WG, Brito JCM, Nizer WSC. Ventilator-associated pneumonia (VAP) caused by carbapenem-resistant Acinetobacter baumannii in patients with COVID-19: two problems, one solution? Med Hypotheses 2020;144:110139.

3. Sharifipour E, Shams S, Esmkhani M, et al. Evaluation of bacterial coinfections of the respiratory tract in COVID-19 patients admitted to ICU. BMC Infect Dis 2020;20:464.

4. Gottesman T, Fedorowsky R, Yerushalmi R, et al. An outbreak of carbapenem-resistant Acinetobacter baumannii in COVID-19 dedicated hospital. Infect Prev Pract 2021;3:100113.

5. Silbert S, Pfaller MA, Hollis RJ, et al. Evaluation of three molecular typing techniques for nonfermentative gram-negative bacilli. Infect Control Hosp Epidemiol 2004;25:847-851.

6. Lee $\mathrm{H}$, Lee $\mathrm{H}$. Clinical and economic evaluation of multidrug-resistant Acinetobacter baumannii colonization in the intensive care unit. Infect Chemother 2016;48:174-180.

7. Weinberg SE, Villedieu A, Bagdasarian N, et al. Control and management of multidrug resistant Acinetobacter baumannii: a review of the evidence and proposal of novel approaches. Infect Prev Pract 2020;2:100077.

8. Saalfeld SMS, Viana GF, Siqueira VLD, et al. Endemic carbapenem-resistant Acinetobacter baumannii in a Brazilian intensive care unit. J Hosp Infect 2009;72:365-368.

9. Moreira RRB, Viana GF, Moraes ACC, et al. Dissemination of Acinetobacter baumannii OXA-23 in old and new intensive care units without transfer of colonized patients. Infect Control Hosp Epidemiol 2018;39:1135-1137.

\title{
Extensive environmental contamination and prolonged severe acute respiratory coronavirus-2 (SARS CoV-2) viability in immunosuppressed recent heart transplant recipients with clinical and virologic benefit with remdesivir
}

\author{
Irina Rajakumar BScPhm, ACPR ${ }^{1}$ (1), Debra L. Isaac MD ${ }^{1,2}$, Nowell M. Fine MD ${ }^{1,2}$, Brian Clarke MD ${ }^{1,2}$, \\ Linda P. Ward $\mathrm{MLT}^{1}$, Rebecca J. Malott PhD², Kanti Pabbaraju MSc ${ }^{4}$, Kara Gill BSc${ }^{4}$, Byron M. Berenger MSc, MD ${ }^{2,4}$, \\ Yi-Chan Lin PhD ${ }^{3}$, David H. Evans PhD ${ }^{3, a}$ and John M. Conly MD ${ }^{1,2, a}$ (i) \\ ${ }^{1}$ Alberta Health Services, Calgary, Alberta, Canada, ${ }^{2}$ University of Calgary, Calgary, Alberta, Canada, ${ }^{3}$ University of Alberta, Edmonton, Alberta, Canada and \\ ${ }^{4}$ Alberta Public Health Laboratory, Alberta Precision Laboratories, Calgary, Alberta, Canada
}

To the Editor-Remdesivir is an antiviral medication that exhibits antiviral activity versus SARS-CoV-2, ${ }^{1-3}$ but in clinical trials, it has demonstrated conflicting results with respect to mortality in patients with severe coronavirus disease 2019 (COVID-19).,5 The use of remdesivir in immunosuppressed patients, including the initial posttransplant period with its high degree of immunosuppression, has not been well studied. ${ }^{6-8}$ We examined the virologic and clinical responses to remdesivir in 2 recent cardiac transplant cases with SARS-CoV-2 infection.

\footnotetext{
Author for correspondence: Dr John Conly, E-mail: john.conly@albertahealthservices. ca or jconly@ucalgary.ca. Or Dr David Evans, E-mail: devans@ualberta.ca

aSenior authors of equal contribution.

Cite this article: Rajakumar I, et al. (2022). Extensive environmental contamination and prolonged severe acute respiratory coronavirus-2 (SARS CoV-2) viability in immunosuppressed recent heart transplant recipients with clinical and virologic benefit with remdesivir. Infection Control \& Hospital Epidemiology, 43: 817-819, https:// doi.org/10.1017/ice.2021.89
}

\section{Methods}

Nasopharyngeal (NP) swabs, saliva, and clinical and environmental samples were collected at regular intervals beginning shortly after admission. They were tested using molecular assays ${ }^{9}$ and quantitative culture (Supplementary Material online). Patients provided informed consent with the approval of the University of Calgary's Health Research Ethics Board (no. 20-0444).

\section{Case descriptions}

Case 1 was a 56-year-old woman with a history of dilated cardiomyopathy with end-stage heart failure, type- 2 diabetes mellitus, hypothyroidism, osteoporosis, and anemia. This patient underwent an orthotopic heart transplant with antithymocyte globulin (ATG) induction and standard triple immunosuppressive therapy. The patient was discharged 30 days later with prednisone, tacrolimus, and mycophenolate mofetil (MMF), and standard prophylactic medications.

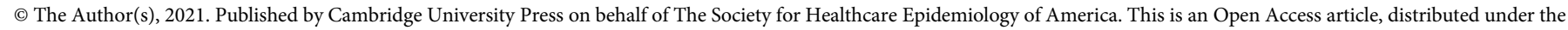
terms of the Creative Commons Attribution licence (http://creativecommons.org/licenses/by/4.0/), which permits unrestricted re-use, distribution, and reproduction in any medium, provided the original work is properly cited. 

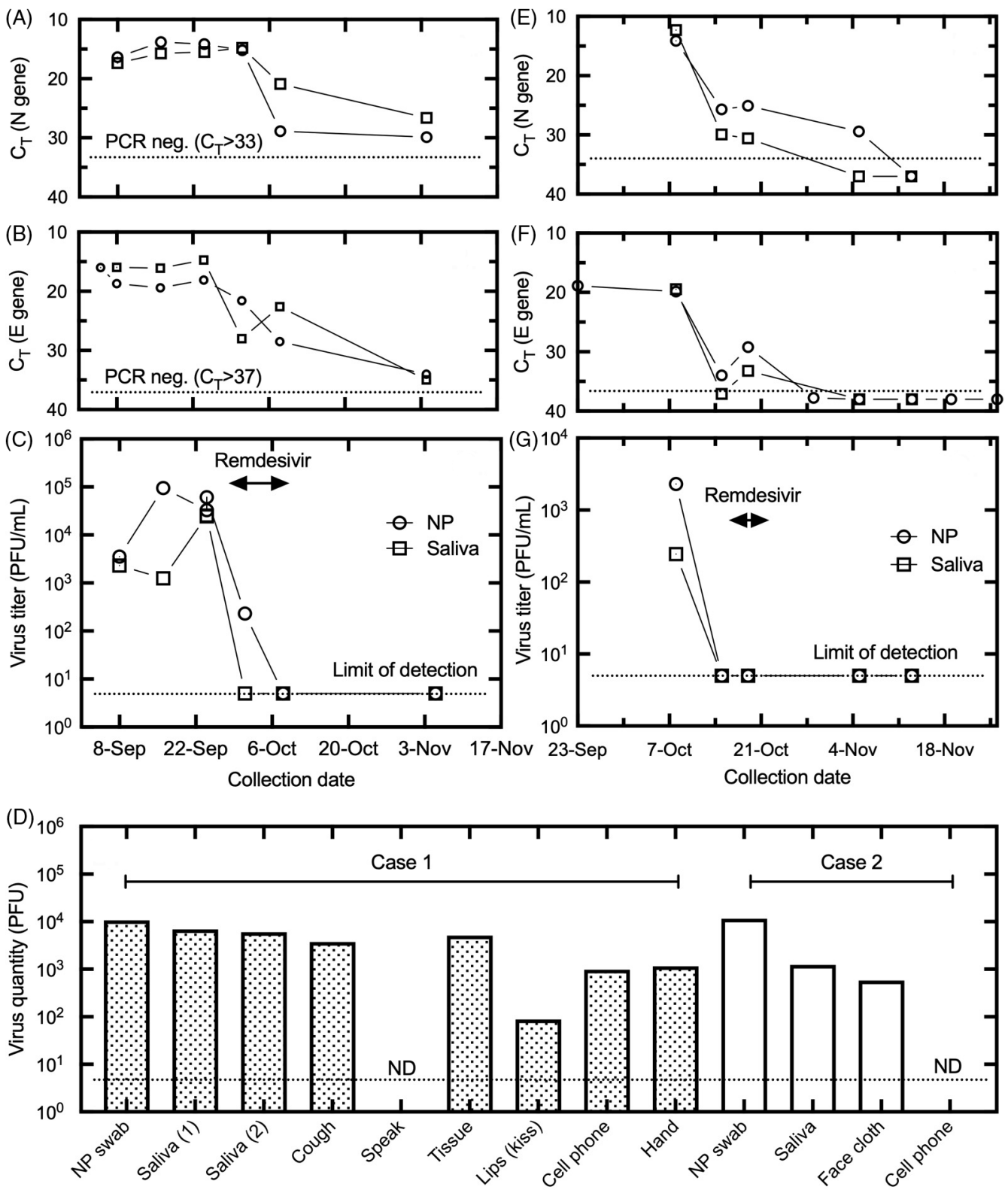

Fig. 1. Results of nasopharyngeal (NP) and saliva specimens collected from cases 1 and 2 from $E$ and $N$ gene RT-PCR assays with cycle threshold (Ct) values (panels $A$ and $B$ plus $\mathrm{E}$ and $\mathrm{F}$, respectively) and quantitative viral culture results on Vero cells (adjusted for volume) from case 1 and case 2 clinical samples (panels $C$ and $\mathrm{G}$, respectively). Culture results on Vero cells are shown for clinical and environmental samples for cases 1 and 2 (panel D). Of 6 environmental samples obtained at the bedside in case 1 (ie, kiss sample, discarded nasal tissues, cell phone, both hands, a cough bag and a 3-5-minute speaking bag), 5 had viable virus; the only exception was the speaking bag. Of 2 environmental samples obtained at the bedside in case 2 (a pledget cut from a face cloth found lying on the patient's bed for at least 1 hour and the cell phone), the face cloth had viable virus whereas the cell phone did not. Dotted lines indicate the Ct value where no samples were positive and the limit of detection for PCR and culture, respectively. Note. PFU, plaque-forming units.

At 5 days after discharge, she was rehospitalized following community exposure to COVID-19. Nasal stuffiness and discharge, sneezing, fatigue, and cough developed on admission day 4 and a NP swab was positive for SARS-CoV-2. All symptoms except fatigue had resolved by day 12 . However, dyspnea, cough, and hypoxia developed on day 15 and the chest radiograph revealed new bibasilar interstitial infiltrates. Corticosteroids and antimicrobials were initiated for presumptive COVID-19 pneumonitis and superimposed bacterial pneumonia, but on day 21 oxygen requirements increased significantly.
Despite discontinuation of MMF and reduction of tacrolimus, cultivatable viral loads increased in the nasopharynx and saliva (Fig. 1), the chest radiograph and clinical condition deteriorated, and mechanical ventilation was considered. Extensive contamination with high quantitative burdens of viable virus was detected in the patient's immediate environment in the hospital room (Fig. 1). A 10-day course of remdesivir was initiated on day 27 (Fig. 1). The patient's clinical condition and chest radiograph improved, allowing oxygen discontinuation by day 32 and discharge on day 44. 
Case 2 was a 33-year-old woman with a history of end-stage heart failure secondary to congenital heart disease, liver cirrhosis, and kidney disease who underwent orthotopic heart transplant with antithymocyte globulin induction therapy. The patient was discharged after 4 weeks on prednisone, tacrolimus, MMF, and standard prophylactic medications.

This patient tested positive for SARS-CoV-2 shortly after discharge and was readmitted 1 week later with progressive dyspnea and hypoxemia requiring intubation. The MMF dose was reduced, and dexamethasone and antimicrobials were initiated. MMF was subsequently held but was later restarted when an echocardiogram demonstrated a reduction in left ventricular systolic function, suggesting acute graft rejection. Significant viable virus burdens were noted in the NP swab and saliva along with a face cloth found on the bed 16 days after initial SARS-CoV-2 positivity (Fig. 1). A 5-day course of remdesivir was initiated followed by clinical recovery and discharge 7 days later.

\section{Discussion}

The first patient's NP and salivary samples (Fig. 1) showed an increase in quantifiable SARS-CoV-2 in the context of the postcardiac transplant immunosuppressive regimen. The initial symptom resolution was not associated with a reduced viable viral load. Extensive contamination with replication- and infectioncompetent SARS-CoV-2 was detected ranging from $10^{2}$ to $10^{4}$ plaque-forming units (PFU) in a kiss sample, discarded nasal tissues, a cell phone, both hands, and cough specimens. The development of presumptive viral pneumonitis correlated with the highest levels of cultivatable virus, and MMF discontinuation had no impact on viral load. The initiation of remdesivir (Fig. 1) was associated with a dramatic decline in virus titers associated with a progressive clinical and radiologic improvement. The samples at day 10 of remdesivir treatment and 23 days after remdesivir revealed no cultivatable virus.

The second patient demonstrated a moderately high viral load (Fig. 1) 18 days after symptom onset. A face cloth found on the bed 16 days after initial SARS-CoV-2 positivity had $\sim 10^{3}$ PFU of replication- and infection-competent virus. Samples obtained immediately prior to remdesivir were $\mathrm{E} / \mathrm{N}$ gene positive but were negative for virus recovery. However, the risk for viral reactivation was considered high given the reintroduction of MMF for early rejection. This patient clinically recovered with repeat specimens obtained at end-of-therapy and 25 days postremdesivir revealing no cultivatable virus.

No published evidence demonstrates that remdesivir offers mortality or graft survival benefits to solid organ transplant (SOT) patients in the immediate posttransplant period. This scenario is likely too uncommon to permit adequately powered demonstration of utility in clinical trials. However, the need to reduce immunosuppressive therapy in SOT patients with COVID-19 creates a competing risk of acute rejection. The risk of graft loss and consequent mortality is substantial, and an agent that can reduce viral loads and permit restoration of immunosuppression can help mitigate that risk, especially in SOT recipients with COVID-19 who are within 6 months of transplantation or following a rejection episode.
These 2 cardiac transplant patients exhibited prolonged viable SARS-CoV-2 carriage from symptom onset, 35 and 26 days, respectively, with rising or unchanged viral loads despite decreasing doses of immunosuppressives in 1 case. Prolonged high viable viral carriage was also reported in hematology-oncology patients. ${ }^{7,10}$ Large quantities of viable SARS-CoV-2 may be shed in highly immunosuppressed patients for prolonged periods of time, creating major clinical and infection control challenges. Our findings of prolonged carriage with extensive environmental contamination adds support for the risk of SARS-CoV-2 infection via the disregarded role of direct contact/fomite transmission, highlighting the need to consider virological countermeasures, which may be a unique role for remdesivir.

Supplementary material. To view supplementary material for this article, please visit https://doi.org/10.1017/ice.2021.89

Acknowledgments. We thank the nursing staff on Unit 64 for their cooperation in the collection of serial specimens in these patients. We thank Gerald Lazarenko and Jeremy Slobodan for facilitating the decision for exceptional access for remdesivir for these 2 patients. We thank the Alberta Precision Laboratory technologists for their assistance with testing and transfer of samples.

Financial support. This publication was funded in part by a grant from Alberta Health Services.

Conflict of interest. The authors declare that they have no competing interests related to this article.

\section{References}

1. Frediansyah A, Nainu F, Dhama K, Mudatsir M, Harapan H. Remdesivir and its antiviral activity against COVID-19: a systematic review. Clin Epidemiol Glob Health 2020. doi: 10.1016/j.cegh.2020.07.011.

2. Gordon CJ, Tchesnokov EP, Woolner E, et al. Remdesivir is a direct-acting antiviral that inhibits RNA-dependent RNA polymerase from severe acute respiratory syndrome coronavirus 2 with high potency. J Biol Chem 2020;295:6785-6797.

3.Williamson BN, Feldmann F, Schwarz B, et al. Clinical benefit of remdesivir in rhesus macaques infected with SARS-CoV-2. Nature 2020; 585:273-276.

4. Pan H, Peto R, Henao-Restrepo AM, et al. Repurposed antiviral drugs for COVID-19-interim WHO solidarity trial results. N Engl J Med 2021;384: 497-511.

5. Beigel JH, Tomashek KM, Dodd LE, et al. Remdesivir for the treatment of COVID-19-Final report. N Engl J Med 2020;383:1813-1826.

6. Baang JH, Smith C, Mirabelli C, et al. Prolonged SARS-CoV-2 replication in an immunocompromised patient. J Infect Dis 2020. doi: 10.1093/infdis/ jiaa666.

7. Decker A, Welzel M, Laubner K, et al. Prolonged SARS-CoV-2 shedding and mild course of COVID-19 in a patient after recent heart transplantation. Am J Transpl 2020;20:3239-3245.

8. Shingare A, Bahadur MM, Raina S. COVID-19 in recent kidney transplant recipients. Am J Transplant 2020;20:3206-3209.

9. Berenger BM, Conly JM, Fonseca K, et al. Saliva collected in universal transport media is an effective, simple and high-volume amenable method to detect SARS-CoV-2. Clin Microbiol Infect 2020. doi: 10.1016/j.cmi.2020. 10.035 .

10. Aydillo T, Gonzalez-Reiche AS, Aslam S, et al. Shedding of viable SARSCoV-2 after immunosuppressive therapy for cancer. N Eng J Med 2020;383: 2586-2588. 